\title{
Characterization of Local Regularity in SAR Imagery by means of Multiscale Techniques: Application to Oil Spill Detection
}

\author{
Marivi Tello $^{(1)}$, Ramon Bonastre ${ }^{(1)}$, Carlos Lopez-Martinez ${ }^{(1)}$, Jordi J. Mallorqui ${ }^{(1)}$, Alessandro Danisi ${ }^{(2)}$, Gerardo Di \\ Martino $^{(2)}$, Antonio Iodice ${ }^{(2)}$, Giuseppe Ruello ${ }^{(2)}$, Daniele Riccio ${ }^{(2)}$ \\ ${ }^{(1)}$ Remote Sensing Laboratory (RSLAB) \\ Universitat Politecnica de Catalunya (UPC) \\ Barcelona, Spain \\ \{marivi.tello, carlos.lopez, mallorqui\}@tsc.upc.edu \\ ${ }^{(2)}$ Department of Electronic and Telecommunication Engineering \\ Università di Napoli "Federico II", Via Claudio 21, 80125 Napoli, Italy.
}

\begin{abstract}
Thanks to their capability to cover large areas, in all weather conditions, during the day as well as during the night, spaceborne Synthetic Aperture Radar (SAR) techniques constitute an extremely promising alternative to traditional surveillance methods. Nevertheless, in order to assure further usability of SAR images, specific data mining tools are still to be developed to provide an efficient automatic interpretation of SAR data. The aim of this paper is to introduce texture analysis performed in the framework of time - frequency theory, as a means to detect oil spills in the sea surface. In particular, an algorithm permitting a precise quantitative characterization of the border between the oil spill candidate and the sea, will allow a novel classification of oil spills and look-alikes.
\end{abstract}

Keywords- wavelet transform; Hölder exponent; multiresolution analysis

\section{INTRODUCTION}

Satellite-based Synthetic Aperture Radar (SAR) sensors are able to provide a powerful surveillance capability, allowing the observation of broad expanses, independently from weather conditions, so much during the day as during the night and without the cooperation of the vessel. With respect to optical imagery, this all time and all weather observation capability remains the most valuable distinctive advantage, but the counterpart is that the interpretation of SAR images, mainly affected by speckle, is quite troublesome. Since a manual treatment is unacceptably slow, unpractical and hardly reproducible, fully or eventually partially computerized schemes are desirable. As a consequence, in order to increase further usability of SAR images, more specific data mining methods are still to be developed to provide efficient tools for automatic interpretation of SAR data.

Accordingly, our purpose is to establish a specific framework for the automatic exploitation of SAR imagery. Inspired by the operation of the brain, the environment proposed is structured following three milestones: spot detection, extraction of linear features and texture analysis. An unsupervised algorithm for spot detection has been previously proposed and intensively validated, mainly in the context of ship detection [1]. Then, another one has recently been introduced for the automatic extraction of linear features [2]. The present paper deals with the last intended issue by proposing a method for the automatic texture analysis of SAR images, mainly in the context of ocean applications.

First, this paper will overview the main drawbacks affecting the automatic oil spill detection in SAR imagery. Then, the use of a multiscale framework will be justified. A method for an unsupervised detection of oil spill candidates, based on texture analysis, will be addressed. Finally, a technique to distinguish oil spills and look-alikes with high reliability will be tested.

\section{A MULTISCALE TIME -FREQUENCY FRAMEWORK FOR} SAR DATA ANALYSIS

\section{A. Particularities of SAR Imagery}

A SAR image $u(x, r)$, where $(x, r)$ are the azimuth and range dimensions respectively, can be modeled as the convolution of the local complex reflectivity of the observed area with the impulse response $u_{0}(x, r)$ of the SAR system. Since a resolution cell is very large in terms of the wavelength of the illuminating electromagnetic wave, the returned echo $\gamma(x, r)$ is the result of the coherent summation of all the returns due to the single scatterers $\gamma_{i}(x, r)$ following a random walk process.

$$
|u(x, r)|=\left|\gamma(x, r) * u_{0}(x, r)\right|=\left|\sum_{i} \gamma_{i}(x, r) * u_{0}(x, r)\right|
$$

Consequently, it is quite obvious that the most appropriate way of facing the interpretation of SAR imagery is by assuming the image samples as realizations of some underlying stochastic process. Then, analysis tools have to be inscribed in a statistical framework.

Each particular application and each particular scenario demands different requirements in the trade-off between space resolution and statistics accuracy. As a consequence, the use of a suitable multiscale time / frequency (space / statistics)

This work was supported by the Spanish MCYT and FEDER funds under project TEC2005-06863-C02-01. 
framework for the interpretation of SAR data is justified as it may deal with this compromise.

\section{B. Oil spills in SAR Images}

The presence of an oil spill in the ocean appears in the SAR image as a dark patch (see Fig. 1). In fact, the viscosity of the pollutant diminishes the roughness of the sea surface and, as a result, the amount of energy backscattered to the radar is reduced. Besides, the SAR signature of an oil spill and its surroundings depends on a number of parameters like wind speed, wave height, and the amount and type of oil released. The shape of the spill will depend on whether the oil was released from a stationary object or from a moving ship, the amount of oil involved and the wind and current history between the release and the image acquisition.

Nevertheless, this dampening effect can also be caused by many different natural and unpredictable phenomena (mainly lack of wind). Hence, the main drawback that unsupervised methods for oil spill detection in SAR imagery have to face is the discrimination of false alarms due to look-alikes (see Fig. 2).
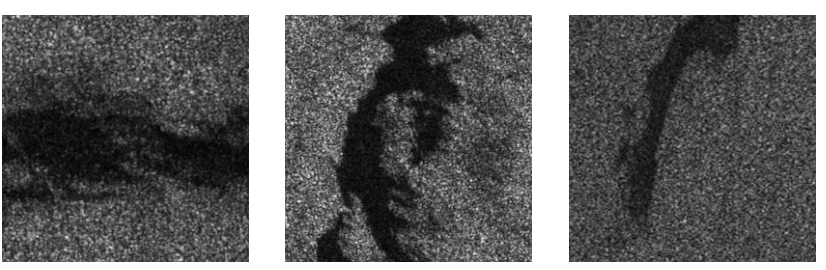

Figure 1. Examples of oil spills in the ocean in SAR imagery.
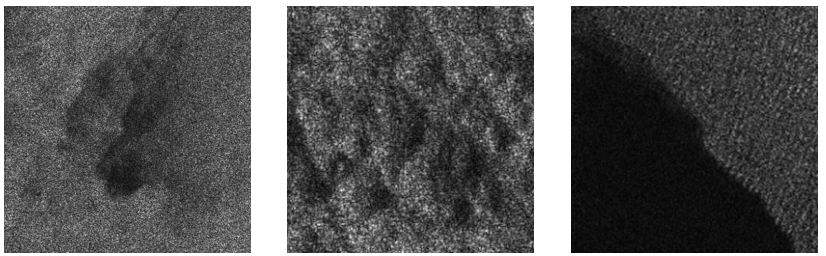

Figure 2. Examples of look-alikes due to marine natural phenomena.

\section{The Wavelet Transform}

Signal processing with wavelets is just one among other time-frequency methods, but it presents clear advantages, mainly in relation to the analysis of transient phenomena. In this section, some of the aspects of the WT will be briefly discussed. Extended details can be found in [3].

Essentially, the WT is the projection of a given complex phenomenon in a function space in which the basic functions or atoms are localized in both time and frequency, coming from dilations and translations of a mother wavelet, $\psi$. In 1D,

$$
W f\left[z, a^{j}\right]=\sum_{m=0}^{N-1} f[m] \psi_{j}^{*}[m-z],
$$

where $z$ is the discrete temporal (or spatial) variable and the parameter $a^{j}$ allows adjusting the temporal (or spatial) duration of the wavelet $\psi_{j}$

$$
\psi_{j}[z]=\frac{1}{\sqrt{a^{j}}} \psi\left[\frac{z}{a^{j}}\right] .
$$

The WT can also be seen as a multiscale differential operator. More specifically, a continuous wavelet $\psi$ has $n$ vanishing moments if and only if there exists a function $\theta$ such that

$$
\psi(t)=(-1)^{n} \frac{d^{n} \theta(t)}{d t^{n}}
$$

As a consequence

$$
W f(u, s)=s^{n} \frac{d^{n}}{d u^{n}}\left(f * \bar{\theta}_{s}\right)(u),
$$

where $s$ is the continuous scale, $u$ is the spatial variable and $\bar{\theta}_{s}$ is the conjugate of the corresponding wavelet at scale $s$, constructed from a dilation of the function $\theta$. Hence, singularities can be detected by finding the abscissa where the wavelet modulus maxima converge at fine scales.

\section{TEXTURE ANALYSIS IN SAR IMAGERY BASED ON THE WT}

\section{A. Theoretical Principles: the Lipschitz regularity}

The objective is to provide a quantitative measure as local as possible and as precise as possible of the regularity of the signal which directly reflects the roughness of the sea surface observed.

By definition, a function $f$ is pointwise Lipschitz $\alpha \geq 0$ at $v$, if there exist $K>0$, and a polynomial $p_{v}$ of degree $m=\lfloor\alpha\rfloor$ such that:

$$
\forall t \in R,\left|f(t)-p_{v}(t)\right| \leq K|t-v|^{\alpha}
$$

If $0 \leq \alpha<1$ then $p_{v}(t)=f(v)$ and the Lipschitz condition becomes:

$$
\forall t \in R,|f(t)-f(v)| \leq K|t-v|^{\alpha}
$$

The decay of the WT amplitude across scales is related to the uniform and pointwise Lipschitz regularity of the signal. Let's suppose a wavelet $\psi$ with $n$ vanishing moments. If $f$ is uniformly Lipschitz $\alpha \leq n$ over $[a, b]$, then there exists $A>0$ such that

$$
\forall(u, s) \in[a, b] \times R^{+},|W f(u, s)| \leq A s^{\alpha+1 / 2}
$$

which is equivalent to

$$
\log _{2}|W f(u, s)| \leq \log _{2} A+\left(\alpha+\frac{1}{2}\right) \log _{2} s
$$

This expression suggests an intuitive method to estimate $\alpha$. The Lipschitz or Hölder exponent at $v$ is thus the maximum slope of $\log _{2}|W f(u, s)|$ as a function of $\log _{2} s$ along the maxima lines converging to $v$. Fig. 3 shows a SAR image of an oceanic scene with a dark region due to a lack of 
wind. The ridges of a vertical cut of this image intercepting the low wind surface are represented. Two distinct groups of ridges can easily be separated, each corresponding to zones exhibiting different textures.
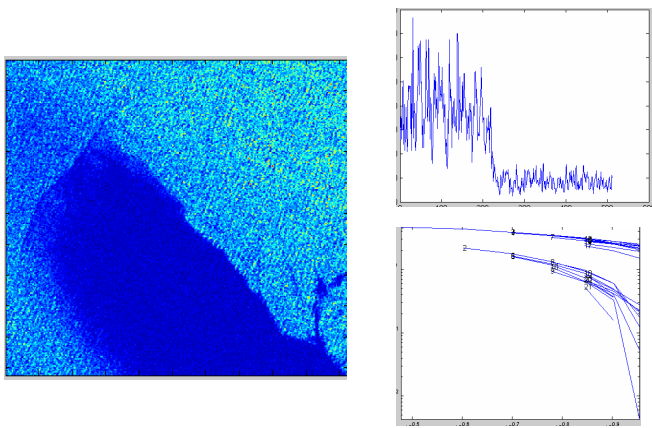

Figure 3. SAR oceanic image with a low wind area (left) (ENVISAT PRI, coast of Barcelona). Vertical cut of the image (top right) and plot of its ridges (bottom right)

\section{B. The Lipschitz Regularity of simulated SAR images}

Willing to work efficiently with images, the definition of Lipschitz exponent has been extended to 2D, by using the 2D continuous WT and considering ridges evolving in 3D (see Fig. 4).
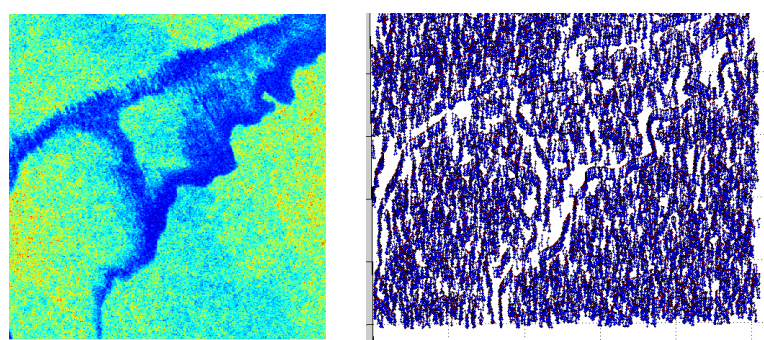

Figure 4. 3D representation of the ridges (right) of a SAR image (left) (ENVISAT ASAR, Prestige oil spill, 2004).

The radiometric level of the sea surface depends strongly on its state and, as a consequence, it is not significant by its own in most oceanic applications. Hence, methods based on direct graylevel thresholding do not provide a sufficiently relevant solution for the automatic interpretation of SAR data. Accordingly, the objective is to build an estimate of the very local spatial variations, independently from the absolute intensity of each individual pixel. In order to check this property, the technique has been tested on a composite simulated image (see Fig. 5). Since, the aim is to estimate the local regularity of the function with independency from the graylevel value, it consists on four replica of the same homogeneous real SAR image, but varying the mean intensity value by multiplying with a constant. Results are also shown in Fig. 5. As stated and desired, the estimation of the local regularity is almost identical in the four different areas of the image. In fact, when superimposed, the histograms of the different regions coincide almost perfectly.
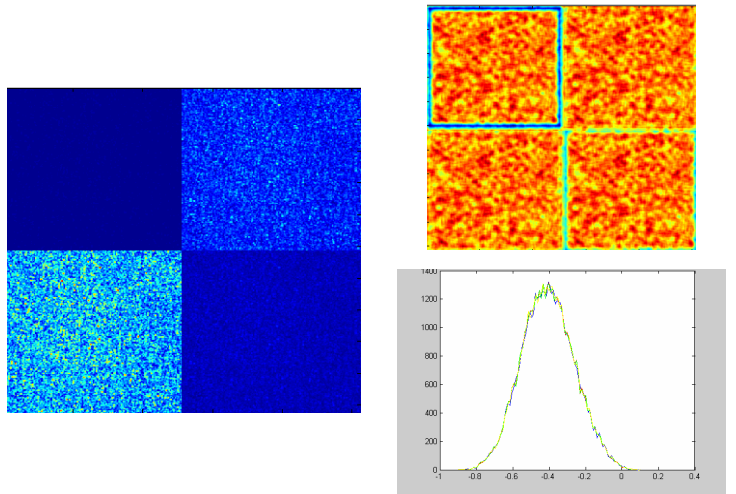

Figure 5. Simulated composite image (left), Lipschitz exponents (top right) and superimposed histograms of the four homogeneous regions (botton right).

Nevertheless, this experiment suggests an intrinsic limitation of this type of analysis, which is the great influence of the borders between homogeneous regions. This effect is due to the cone of influence of the wavelet used which reflects the presence of an important discontinuity. The cone of influence hides a symmetric region on the vicinity of the irregularity becoming wider when the number of scales involved increases. Therefore, the effects of the cone of influence can be reduced by using less scales, but at the expense of estimations much more noisy.

A second experiment is carried out by using an image produced by a SAR simulator [4] (see Fig. 6). This simulation exhibits two dark homogeneous patches in a calm sea surface. Even if both of them have the same damping ratio, the one in the upper right zone has been modeled with the parameters corresponding to an artificial oil spill and the one in the bottom left with those corresponding to a lack of wind surface. The Hölder exponents of this image have been computed. In order to minimize the effects of the cone of influence in the borders, just a few scales have been taken into account. Even if the result appears to be noisy, it can be observed that the method used is sensitive enough to discriminate an oil spill from a look-alike, even with the same mean intensity, in a simulated homogeneous image.
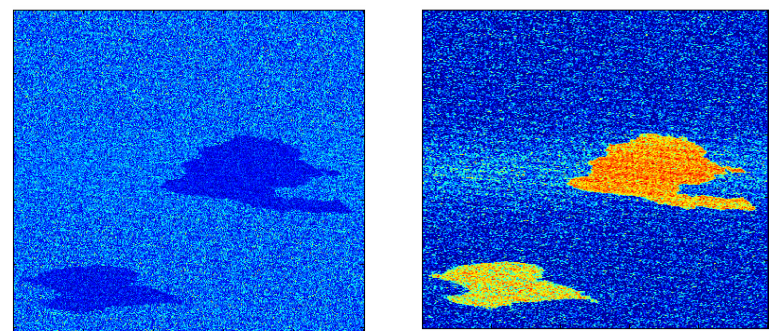

Figure 6. Lipschitz exponents with a small number of scales(right) of a simulated image (left) with two dark patches (oil spill and look alike).

Unfortunately, real scenarios are more heterogeneous and, since oil spills tend to be elongated and narrow, the effect of the cone of influence usually disables the measure of a precise texture estimate inside of it. Nevertheless, a collateral effect to be taken into account is that the technique is extremely sensitive to the presence of a transition. Moreover, a relevant distinctive characteristic between low wind areas and oil spills 
relies on the type of discontinuity. The passing from an oil free sea surface to a polluted one is more abrupt than that produced by the presence of a natural phenomenon. Based on this premises, a method pursuing a reliable and unsupervised discrimination between oil spills and look alikes is introduced in the next section.

\section{AUTOMATIC DISCRIMINATION OF OIL SPILLS AND LOOK ALIKES}

The Hölder exponents of a set of RADARSAT and ENVISAT oceanic images have been computed. Since the objective is to provide a reliable quantitative measure of the irregularity of the contour, we are less affected by the cone of influence and a higher number of scales can be involved. Moreover, the measure of the Hölder exponents is restricted to longest chains in order to reduce noise in the final representation. Results are depicted in Figs. 7 and 8. On the one hand, the capability of this estimate to precisely measure the transition between two textures is confirmed. Furthermore, a different behavior is observed between discontinuities due to the presence of oil spills (see Fig. 7) and those due to natural features (see Fig. 8) which tend to be smoother.
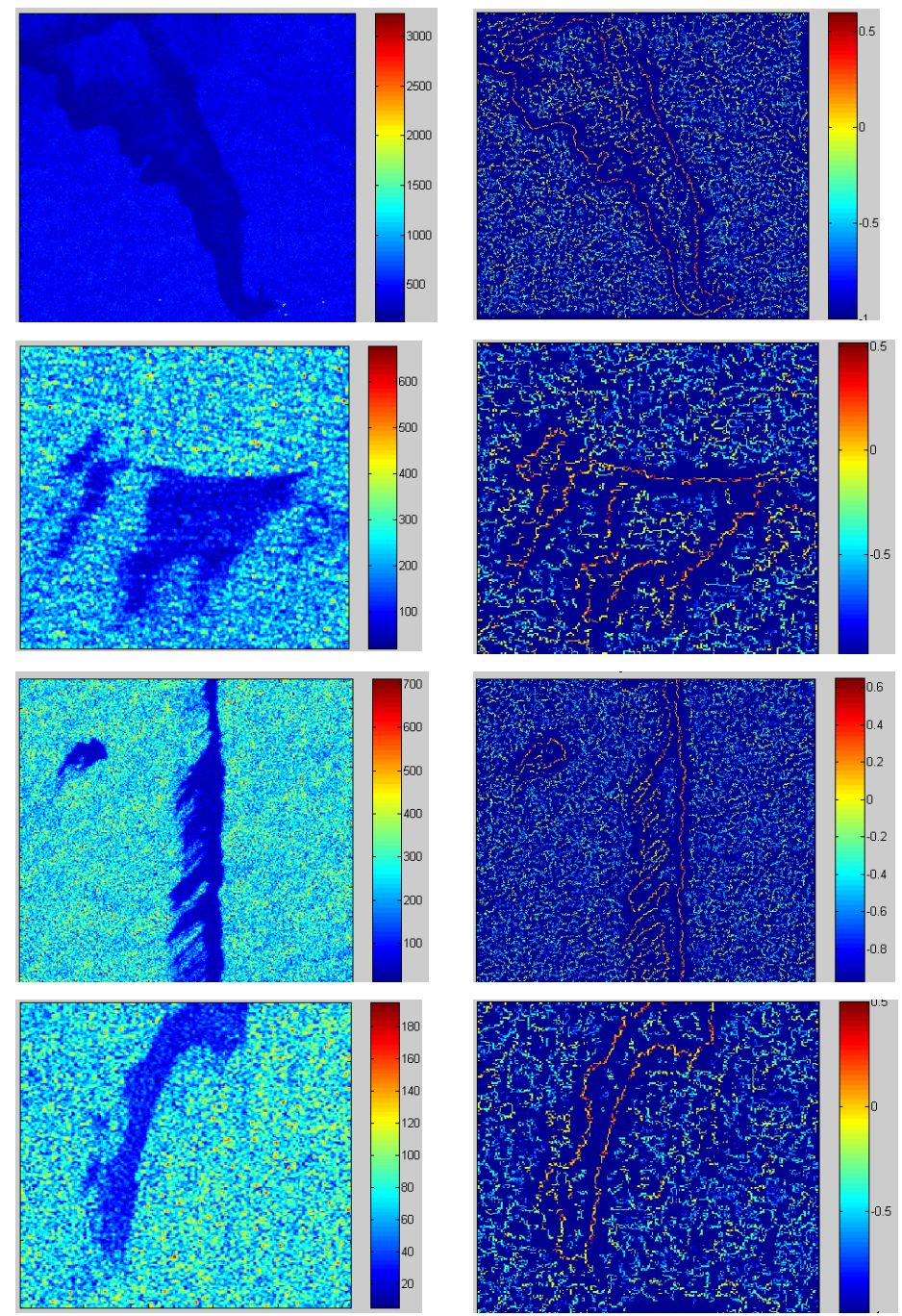

Figure 7. Hölder exponents (right column) of different ENVISAT SAR scenes in the presence of oil spills (left column).
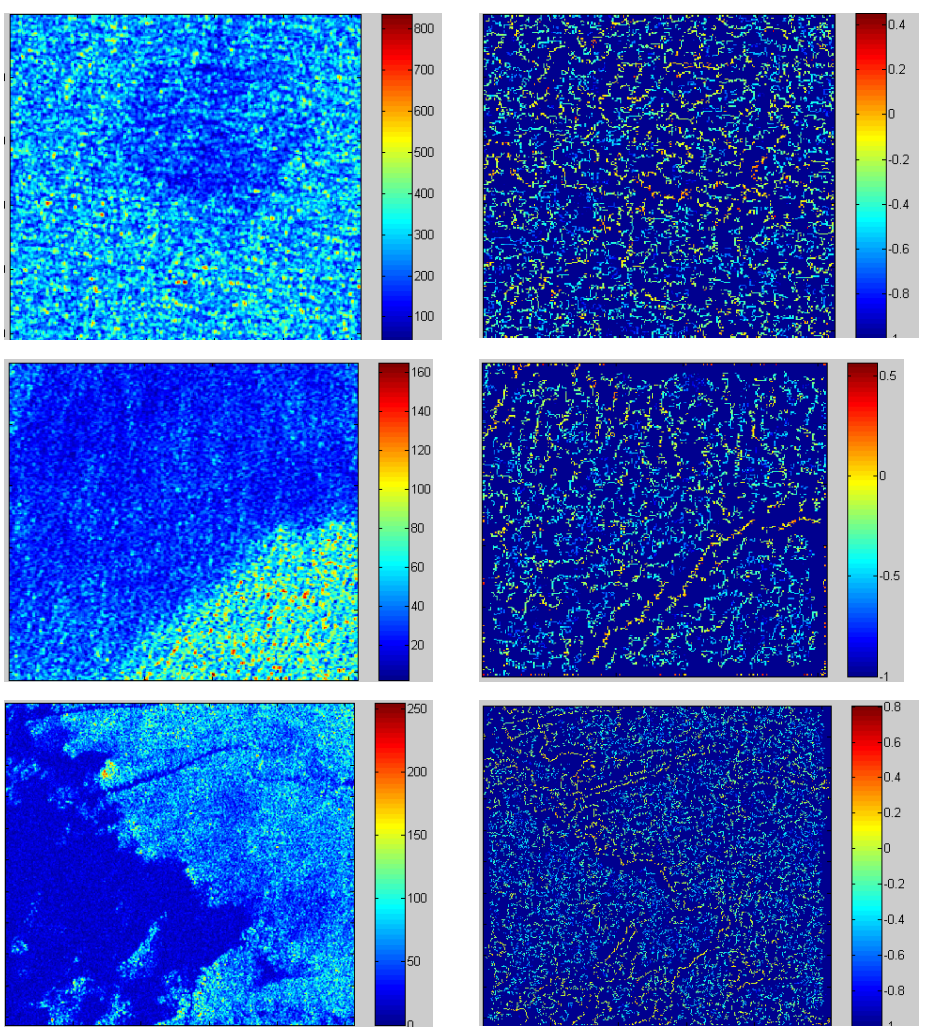

Figure 8. Hölder exponents (right column) of different SAR scenes in the presence of look alikes.

\section{CONCLUSION}

A framework for the unsupervised interpretation of SAR imagery, based on the multiscale time - frequency theory, has been proposed in this paper. In particular, automatic oil spill detection in the ocean has been addressed. After reviewing the main drawbacks, the Hölder exponent has been introduced as a measure of the local regularity of the signal. It has been shown that the presence of a cone of influence disables a precise estimation of the texture inside the spill. Hence, it has been suggested to use the different type of transition between the oil free sea surface to the polluted one and to the dark area due to natural features.

\section{REFERENCES}

[1] Tello, M.; López-Martínez, C.; Mallorqui, J. Automatic Vessel Monitoring with Single and Multidimensional SAR Images in the Wavelet Domain, International Society for Photogrammetry and Remote Sensing

[2] Tello, M.; López-Martínez, C..; Mallorqui, J. Automatic Extraction of Linear Features in SAR Images by means of the Wavelet Transform, Proceedings on ENVISAT Symposium 2007.

[3] Mallat, A Wavelet Tour on Signal Processing, Academic Press, 1999.

[4] Danisi, A. et al. SAR Simulation of Ocean Scenes Covered by Oil Slicks with Arbitrary Shapes, Proceedings on IGARSS 2007. 